\title{
Experimental Investigation of Pulse Modulation Schemes in Free Space Optical Communication Under Turbulence
}

\author{
Y.P. Arul Teen ${ }^{\mathrm{a}, 1}$, NimmyLazer ${ }^{\mathrm{b}}$, J.M. Aravind ${ }^{\mathrm{c}}$ and Rajesh K. B. ${ }^{\mathrm{d}}$ \\ ${ }^{a}$ Department of ECE, University College of Engineering, Nagercoil, India \\ ${ }^{\mathrm{b}}$ Department of ECE, Marthandam College of Engineering \& Technology, \\ Tamilnadu, India \\ 'Department of ECE, Mar Ephraem College of Engineering \& Technology, \\ Tamilnadu, India \\ ${ }^{\mathrm{d} D e p a r t m e n t ~ o f ~ P h y s i c s, ~ C h i k k a n n a ~ G o v e r n m e n t ~ A r t s ~ C o l l e g e, ~ T i r u p u r, ~ I n d i a ~}$
}

\begin{abstract}
When we allow an optical beam to travel through Free Space Optical (FSO) Communication system, the optical beam faces immense issues due to so many factors such as diffraction, scattering, signal absorption, etc. These factors put down the quality rate of FSO communication system. In this experimental analysis, an artificial controlled turbulence chamber was built to create FSO communication with optical source laser. The signal to be tested is generated with the help of a LASER source, is allowed to pass on through the immense turbulent states by employing various pulse modulation schemes such as Pulse Amplitude Modulation (PAM), Pulse Duration Modulation (PDM), and Pulse Position Modulation (PPM) separately. In all the three modulation schemes, the parameters such as phase Jitter and $3 \mathrm{~dB}$ bandwidth are observed and the observed values are compared. The results show that phase jitter value is 9.8414 radians for PPM. Also, PPM has the highest ability to overcome jitter than the remaining two schemes under high atmospheric turbulence parameters. However, in the case of Bandwidth utilization, PDM modulation reduces its utilization to $12 \mathrm{MHz}$.
\end{abstract}

Keywords. Free Space Optical communication; PPM; PAM; PDM; Phase Jitter; $3 \mathrm{~dB}$ bandwidth, performance, measurement.

\section{Introduction}

In the current scenario, free space optical (FSO) communication focuses a deep attentiveness in optical community because of its immeasurable advantages such as huge amount of data transmission over unlicensed spectrum, wireless communication technique used to send and receive various types of data at faster rate, larger bandwidth, gives high performance in optical networks, etc. [7-12]. Although, along with the advantages FSO faces certain troubles due to the presence variety of turbulent factors present in the environment which effects its performance [1,13]. FSO chooses Laser as

${ }^{1}$ Y.P. Arul Teen, Dept of ECE, University College of Engineering, Nagercoil, TamilNadu, India.

Email: arulteen@yahoo.co.in 
an optical source compared to other sources, but the transmitted signal over atmosphere get distorted due to turbulence. Experimental demonstration of OOK and BPSK-SIM modulation schemes on scintillation in fixing the threshold value on an FSO system is shown in $[2,14]$. The presence of turbulence causes error in the received data [13]. By properly observing the jitter values the degree of deviation of the received signal from the actual transmitted can be found out clearly. A variety of statistical models were used for studying the impact of pointing errors on FSO communication systems [5-6]. By using PPM, a good channel capacity can be achieved in an optical link [15]. In PAM, data is transmitted by varying the amplitudes of the individual pulses. In PDM, long pulses expend considerable power while bearing no additional information [18]. Here, phase jitter, time jitter, and $3 \mathrm{~dB}$ parameters are used for FSO analysis experimentally by using various modulation schemes for propagating the data in a specially designed artificial turbulence chamber for FSO communication and the outputs are compared. Description of problem and FSO test bench are discussed in section 2. The results and discussion is highlighted in section 3 and section 4 deals with conclusion [19-21].

\section{Problem Description and FSO Test Bench}

The quality of transmitted data can be degraded due to the fluctuation in phase and intensity of laser beam $[14,18]$. The range of FSO link, atmospheric attenuation and pointing error under various weather conditions have been studied [4]. Compensation of phase fluctuation is very essential to achieve a very high data rate. Scintillation, beam spreading and beam wandering are some other problems that affects laser beam [17]. Therefore, proper studies and compensation techniques are important so that the reliability of data transmission through FSO can be increased.

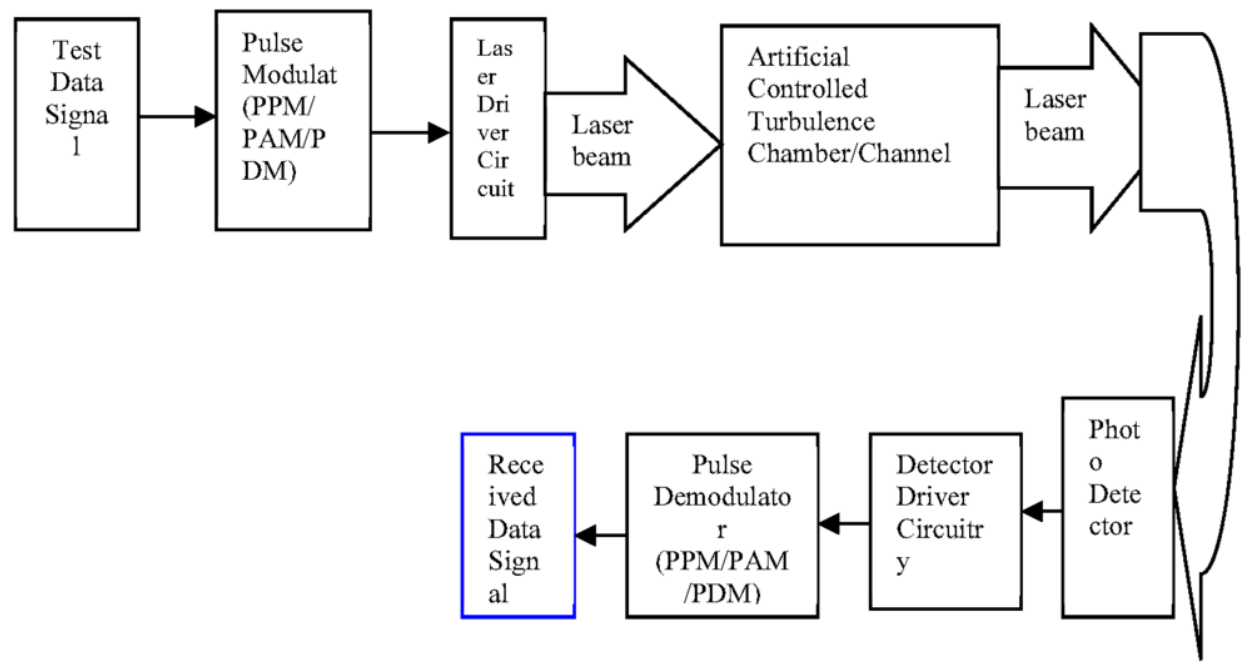

Figure 1.Free Space Optical Communication Test Bench Experimental Setup

The test bench setup used for experimenting FSO system is shown in Figure 1, which consists of a transmitter-receiver for transmission and receiving data, and channel for communicating. In the transmitter section, signal generator is used to generate test 
data. The modulating signal frequency is $4 \mathrm{KHz}$. The modulation scheme may be either PPM or PAM or PDM. The laser source is driven by the modulated signal and the wavelength of laser ranges between $650-680 \mathrm{~nm}$. The test bench maintains line of sight between transmitter and receiver ends. The separation between both ends are maintained as $125 \mathrm{~cm}$ throughout the experiment. The optical beam after modulation travels through the turbulence chamber. The turbulence chamber is artificially filled with various atmospheric turbulence components such as smoke and fog. The LASER beam affected by the various turbulence inside the chamber are allowed to receive by the PIN photo detector. By applying the demodulation in a proper manner, we can measure the phase and time jitter of the received signal with the help of a spectrum analyzer. The experiment is repeated for each pulse modulation technique namely PPM, PAM, and PDM. Turbulence chamber dimension is $125 \times 25 \times 25 \mathrm{~cm}^{3}$. The temperature inside the chamber is regulated in such a way that it will always maintains 45 degrees and also, from the beginning till the end of the experiment turbulence conditions were maintained constant.

\section{Results and Discussion}

The FSO system explained above is tested experimentally by the proposed artificial turbulence chamber. In this experiment, the frequency spectrum with center frequency is $1.5 \mathrm{GHz}$ and span of frequency is $3 \mathrm{GHz}$ are considered. First, the system is tested by giving test data as input to different modulation scheme (PAM, PDM, PPM) under without turbulence. The output readings are taken using standard spectrum analyzer, which is connected with the demodulation kit. Figure 2 shows the phase and time jitter measurement using spectrum analyzer for different modulation scheme without turbulence. The phase and time jitter measurement in PAM with no turbulence is shown in figure 2(a) and the output shows that 9.8598 radians and $1.0955 \mathrm{nsec}$ is the value for phase and time jitter. Same analysis method and modulation scheme is employed in PDM with no turbulence and figure 2 (b) represents the result. It is monitored that the 9.854 radians and $859.55 \mathrm{psec}$ is the value for phase and time jitter. When using PPM, 7.7359 radians and $1.0972 \mathrm{nsec}$ is the value for phase and time jitter, which is shown in figure 2 (c).

(a) PAM

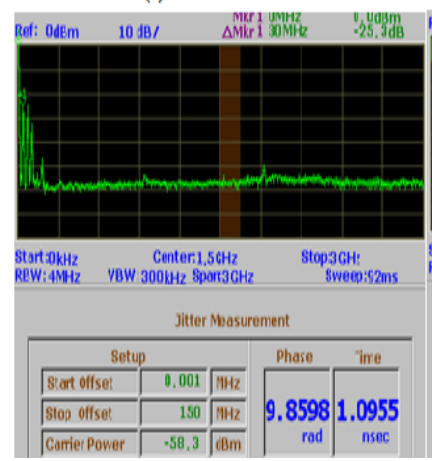

(b) PIVM

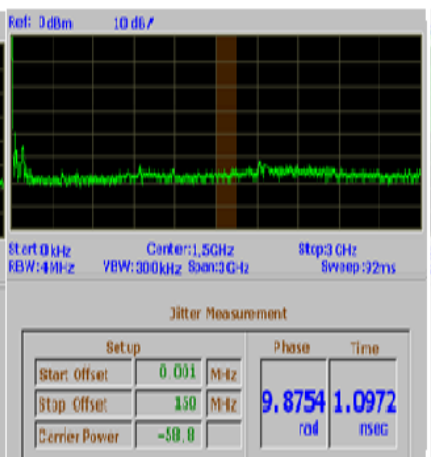

(c) PPM

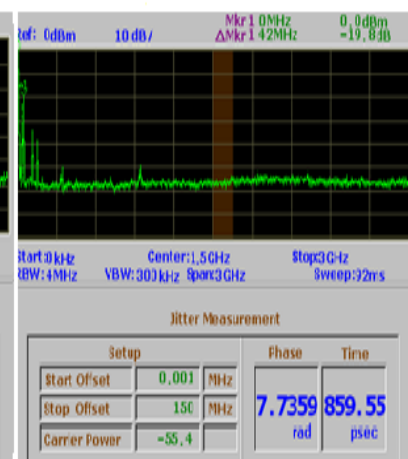

Figure 2. Phase and time jitter without turbulence, a) PAM b) PDM c) PPM

Figure 3 represents the phase and time jitter measurement using spectrum analyzer for different modulation scheme with turbulence. The phase and time jitter measurement in PAM with turbulence is shown in figure 3(a) and it is monitored that 10.445 radians and 
$1.1605 \mathrm{nsec}$ is the value for phase and time jitter. Same procedure is done using PDM in the presence of turbulence, which is shown in figure 3 (b), the observed value is 10.195 radians and $1.1329 \mathrm{psec}$ for phase and time jitter. When using PPM modulation, the noted phase jitter is 9.8414 radians and time jitter is $1.0934 \mathrm{nsec}$ and it is representing in figure 3 (c). Next, bandwidth utilization is analyzed experimentally for all the different three modulation schemes without and with turbulence.

(a) PAM

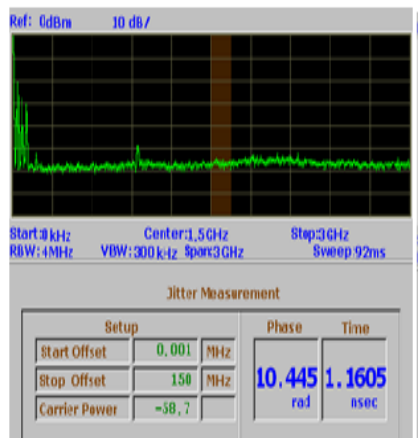

(b) PWM

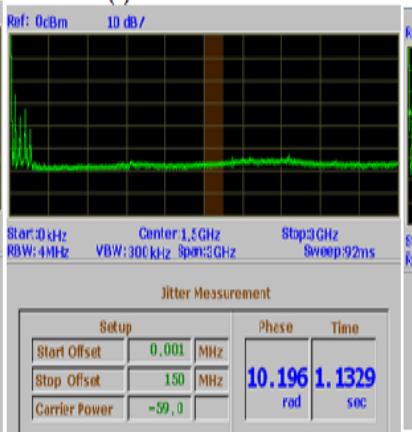

(c) PPMI

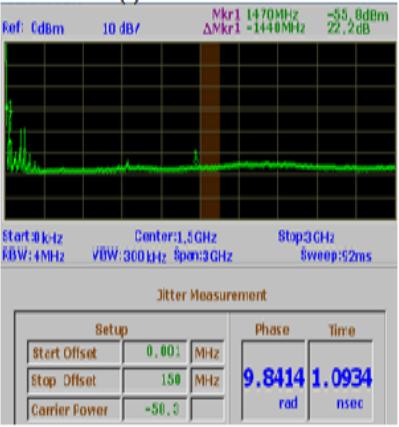

Figure 3. Phase and time jitter with turbulence, a) PAM b) PDM c) PPM

The $3 \mathrm{~dB}$ measurement of bandwidth utilization is measured using spectrum analyzer for different modulation scheme under without turbulence conditions. The $3 \mathrm{~dB}$ bandwidth measurement using PAM without turbulence is observed as $30 \mathrm{MHz}$. The same method applied for PDM and PPM under without turbulence, the bandwidth is $30 \mathrm{MHz}$ for PDM and $12 \mathrm{MHz}$ for PPM. The $3 \mathrm{~dB}$ measurement of bandwidth utilization with turbulence condition shows that using PAM, the bandwidth is 30MHz. For PDM and PPM under turbulence conditions bandwidth is $12 \mathrm{MHz}$ and $30 \mathrm{MHz}$. The experimental results of different pulse modulation techniques with and without turbulence of phase and time jitter is shown in table 1 . In which it is clearly shows the phase jitter is very low in PPM when compared with PDM and PAM under with and without turbulence conditions. From this it is clearly analyzed and verified that the huge amount of data transmission over unlicensed spectrum, wireless communication technique with high performance computing will send and receive various types of data at faster rate, larger bandwidth, gives high performance in optical networks, etc. Therefore, in optical high performance networks, using laser as optical source and selecting PPM modulation scheme for modulating laser source, we can achieve high data transmission rate with less jitter.

Table 1.Comparison of Modulation schemes

\begin{tabular}{|c|c|c|c|c|c|c|}
\hline \multirow[b]{2}{*}{ Parameter } & \multicolumn{2}{|l|}{ PAM } & \multicolumn{2}{|l|}{ PDM } & \multicolumn{2}{|l|}{ PPM } \\
\hline & $\begin{array}{l}\text { Without } \\
\text { Turbulenc } \\
\text { e }\end{array}$ & $\begin{array}{l}\text { With } \\
\text { Turbulenc } \\
\text { e }\end{array}$ & $\begin{array}{l}\text { Without } \\
\text { Turbulenc } \\
\text { e }\end{array}$ & $\begin{array}{l}\text { With } \\
\text { Turbulenc } \\
\text { e }\end{array}$ & $\begin{array}{l}\text { Without } \\
\text { Turbulence }\end{array}$ & $\begin{array}{l}\text { With } \\
\text { Turbulenc } \\
\text { e }\end{array}$ \\
\hline $\begin{array}{l}\text { Phase } \\
\text { Jitter }\end{array}$ & $9.8598 \mathrm{rad}$ & $10.445 \mathrm{rad}$ & $9.8754 \mathrm{rad}$ & 10.196rad & 7.7359rad & $9.8414 \mathrm{rad}$ \\
\hline $\begin{array}{l}\text { Time } \\
\text { Jitter } \\
\text { 3dB }\end{array}$ & $\begin{array}{l}1.09555 \mathrm{nse} \\
\mathrm{c}\end{array}$ & $1.1605 \mathrm{nsec}$ & $1.0972 \mathrm{nsec}$ & $1.1329 \mathrm{sec}$ & $\begin{array}{l}859.55 \text { picose } \\
\text { c }\end{array}$ & $1.0934 \mathrm{nsec}$ \\
\hline $\begin{array}{l}\text { Bandwidt } \\
\text { h }\end{array}$ & $30 \mathrm{MHz}$ & $30 \mathrm{MHz}$ & $30 \mathrm{MHz}$ & $12 \mathrm{MHz}$ & $12 \mathrm{MHz}$ & $30 \mathrm{MHz}$ \\
\hline
\end{tabular}




\section{Conclusion}

It is apparent from the results that over dense atmospheric turbulence, the signal strength immunity level of PDM and PAM are very low than that of PPM. Very high phase jitters are observed in PDM and PAM making them in efficient modulation techniques for consistent data transmission over FSO. Results shows that PPM is the best modulation technique based on Jitter analysis. In the point of view of bandwidth utilization, PDM is the best choice than PPM, but in the case of phase and time fluctuation, PPM is considerable modulation choice. Therefore, in optical high performance networks, using laser as optical source and selecting PPM modulation scheme for modulating laser source, we can achieve high data transmission rate with less jitter. Work is currently in progress to increase the length of channel and by applying other digital modulation techniques to identify Jitter performance.

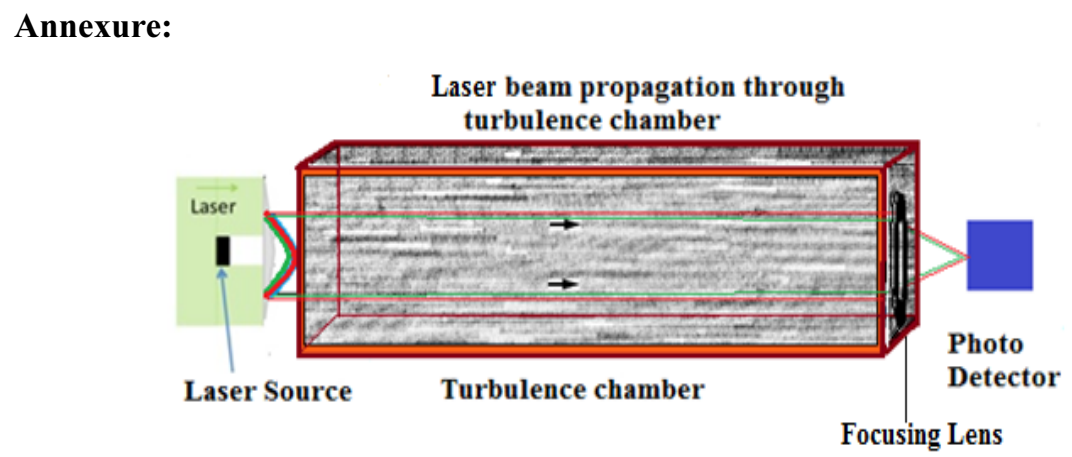

Figure 4.Laboratory FSO experimental setup

Table 2. Experimental System Parameters

\begin{tabular}{|c|c|c|c|}
\hline Laser Source & Turbulence Chamber & Focusing Lens & $\begin{array}{l}\text { Photo } \\
\text { Diode }\end{array}$ \\
\hline $\begin{array}{l}\text { Laser color: RED } \\
\text { Wavelength: } 650 \mathrm{~nm} \pm 10 \\
\text { Laser max. output power: } \\
3 \mathrm{~mW} \\
\text { Grade: Class IIIA(Betatx) } \\
\text { Size }: 65 \times 14 \times 14 \mathrm{~mm} .\end{array}$ & $\begin{array}{l}\text { Dimension: } 125 \times 25 \times 25 \mathrm{~cm}^{3} \\
\text { Artificial Turbulence: Fog } \\
\text { and Smoke } \\
\text { Condition: } 45^{\circ} \mathrm{C}\end{array}$ & $\begin{array}{l}\text { Type: Convex type } \\
\text { Material=UV fused silica with } \\
\text { refractive index value } \mathrm{n} \text { is } \\
1.4585 \text {. } \\
\text { Lens diameter }=12.5 \mathrm{~mm} \text {. } \\
\text { Edge thickness }=4 \mathrm{~mm} \text {. } \\
\text { Focal length }: 13.1 \mathrm{~mm}\end{array}$ & $\begin{array}{l}\text { Silicon } \\
\text { NPN } \\
\text { type } \\
: 2 \mathrm{~N} 5777\end{array}$ \\
\hline
\end{tabular}

\section{References}

[1] Chaleshtory, Z.N., Gholami. A., Ghassemlooy. Z., and Sedghi. M. Experimental investigation of environment effects on the FSO link with turbulence. IEEE Photonics Technol. Lett. 2017; 29(17): 1435-1438

[2] Dabiri. M.T. and Sadough. S.M.S. Generalized blind detection of OOK modulation for free-space optical communication. IEEE Commun. Lett. 2017; 21(10): 2170-2173

[3] Ytian,,Tianwen, Shuang,Shijie,Huibin. Timing jitter's influence on the symbol error rate performance of the L-ary pulse position modulation free-space optical link in atmospheric turbulent channels with pointing errors.Journal of Optical Engineering. Mar 2017; 56(3): 036116-1-3.

[4] Adela.M, Seleem, Nasr, El-Khobby. Transmission of 128Gb/s Optical QPSK Signal over FSO Channel under Different Weather Conditions and Pointing Errors. IOP Publishing-Journal of Physics: Conference Series. 2020; 1447 -012055: 1-7 
[5] Ruben. B.R, Antonio. G.R, Carmen. C.V, and Beatriz. C.V. Novel approximation of misalignment fading modeled by Beckmann distribution on free-space optical links.OPTICS EXPRESS. Oct 2016; 24(20): 22635-22649.

[6] Roumelas, Nistazakis, Leitgeb, Stassinakis, Tombras.On the performance of optical wireless communication links impaired by time jitter, M-turbulence and pointing errors.Journal of Optics Communication. Apr 2020; 472 (126033): 1-8.

[7] Andrews L,Philips R.L, and Hopen C.Y.Laser Beam Scintillation with Applications.SPIE Press. 2001.

[8] TsiftisT.A,Sandlidis.H.G,KaragiannidisG.K.SagiasN.C. Multihopfree-spaceoptical communications over strong turbulence channels.IEEE ICC Proc. June 2006; 2755-2759.

[9] Gagliardi.R and Karp.S .Optical Communications. Wiley. 1995.

[10] Willebrand H and Ghuman.B.S. Free Space Optics: Enabling Optical Connectivity in Today's Network. IN: SAMS. 2002.

[11] Safari M and Uysal M. Relay-assisted free-space optical communication.IEEE Transactions on Wireless Communications. Dec 2008; 7(12): 5441 - 5449.

[12] Theodoros. A. Tsiftsis, Harilaos G. Sandalidis, George K. Karagiannidis, and Murat Uysal. OpticalWirelessLinkswithSpatialDiversityoverStrongAtmosphericTurbulence Channels. IEEE Transactions On Wireless Communications. Feb 2009; 8(2): 951-957

[13] Deva K. Borah, Member, and David G. Voelz.Pointing Error Effects on Free-Space OpticalCommunicationLinksinthePresenceofAtmosphericTurbulence.JournalOf Light wave Technology. Sept 2009; 27(18): 3965-3973

[14] Popoola WO and Ghassemlooy Z. Scintillation effect on intensity modulated laser communication systems - a laboratory demonstration. Journal Of Optics \& Laser Technology. June 2010; 42: 682-692

[15] Peppas KP and Datsikas CK. Average Symbol Error Probability of General-Order Rectangular Quadrature Amplitude Modulation of Optical Wireless Communication Systems Over Atmospheric Turbulence Channels. 102 J. Opt. Commun.Netw. Feb 2010; 2(2): 102-110.

[16] LatsaBabuPedireddi and BalajiSrinivasan.Characterization of Atmospheric Turbu- lence Effects and their Mitigation Using Wavelet-Based Signal Processing. IEEE Transactions On Communications. June 2010; 58(6): 1795-1802

[17] Majumdar AK. Free-space laser communication performance in the atmospheric channel. J. Opt. and Fiber Commun. Research.2005; 2: 345-396

[18] Simon Haykin. Communication Systems.4th Edition; Wiley Publications.2004.

[19] Demir, Alper. Phase noise and timing jitter in oscillators with colored-noise sources. IEEE Transactions on Circuits and Systems I: Fundamental Theory and Applications. Dec 2002; 49(12): 1782-1791.

[20] V. D. AK, S. Sharmila, Abhishek Kumar, A. K. Bashir, Mamoon Rashid, Sachin Kumar Gupta \&Waleed S. Alnumay . novel solution for finding postpartum haemorrhage using fuzzy neural techniques.Neural Computing and Applications (2021) (https://doi.org/10.1007/s00521-020-05683-z)

[21] R. Subha Shini et.al., “ Recurrent Neural Network based Text Summarization Techniques by Word Sequence Generation”, IEEE International Conference on Inventive Computation Technologies (ICICT), 2021, DOI: 10.1109/ICICT50816.2021.9358764 